\title{
The Effect of Coupled Dark Energy on the Alignment between Dark Matter and Galaxy Distributions in Clusters
}

\author{
Marco Baldi ${ }^{1,2}$, Jounghun Lee ${ }^{3}$ and Andrea V. Macciò ${ }^{4}$
}

\begin{abstract}
We investigate the effects of a coupled Dark Energy (cDE) scalar field on the alignment between satellites and matter distributions in galaxy clusters. Using high-resolution N-body simulations for $\Lambda$ CDM and cDE cosmological models, we compute the probability density distribution for the alignment angle between the satellite galaxies and underlying matter distributions, finding a difference between the two scenarios. With respect to $\Lambda$ CDM, in cDE cosmologies the satellite galaxies are less preferentially located along the major axis of the matter distribution, possibly reducing the tension with observational data. A physical explanation is that the coupling between dark matter and dark energy acts as an additional tidal force on the satellite galaxies diminishing the alignments between their distribution and the matter one. Through a Wald test based on the generalized $\chi^{2}$ statistics, the null hypothesis that the two probability distributions come from the same parent population is rejected at the $99 \%$ confidence level. It is concluded that the galaxy-matter alignment in clusters may provide a unique probe of dark sector interactions as well as the nature of dark energy.
\end{abstract}

Subject headings: cosmology:theory — large-scale structure of Universe

\section{INTRODUCTION}

The concordance cosmological model - based on a family of non-relativistic massive particles possibly weakly interacting with the standard sector of particle physics (Cold Dark

\footnotetext{
${ }^{1}$ Excellence Cluster Universe, Boltzmannstr. 2, D-85748 Garching, Germany ; marco.baldi@universecluster.de

${ }^{2}$ University Observatory, Ludwig-Maximillians University Munich, Scheinerstr. 1, D-81679 Munich, Germany

${ }^{3}$ Astronomy Program, Department of Physics and Astronomy, FPRD, Seoul National University, Seoul 151-747, Korea ; jounghun@astro.snu.ac.kr

${ }^{4}$ Max-Planck-Institut für Astronomie, Königstuhl 17, 69117 Heidelberg, Germany ; maccio@mpia.de
} 
Matter particles, CDM hereafter) and on a cosmological constant $\Lambda$ - very successfully accounts for a large wealth of observational data, but poses significant theoretical puzzles and requires an extreme fine-tuning of its basic parameters. In this context, alternative scenarios have been explored, as i.e. the possibility that the cosmological constant $\Lambda$ be replaced by a Dark Energy (DE) component represented by a classical dynamical scalar field $\phi$ evolving in a self interaction potential (Wetterich 1988; Ratra \& Peebles 1988). A further step in the exploration of alternative cosmological models has been recently proposed, speculating about a possible direct interaction between the DE and the CDM sectors of the Universe (Wetterich 1995; Amendola 2000; Amendola 2004). Such coupled Dark Energy models (cDE) have been widely investigated in recent years concerning their background evolution and their effects on structure formation (e.g. by Mainini \& Bonometto; Pettorino \& Baccigalupi 2008; Abdalla et al. 2009; Wintergerst \& Pettorino 2010) by means of specifically designed N-body algorithms aimed at exploring the nonlinear evolution of cosmic structures within these alternative scenarios (Macciò et al. 2004; Baldi et al. 2010; Baldi 2010; Li \& Barrow 2010). These studies have led to the highlight of some distinctive features in the evolved matter density fields of cDE models and have shown how the properties of nonlinear collapsed objects can be significantly affected by the dark interactions in a potentially observable way (Baldi \& Viel 2010; Baldi \& Pettorino 2010). Therefore, one of the most important tasks in the present investigation of these alternative models consists in linking the predictions obtained for the CDM density distribution to directly observable quantities.

Lee (2010) very recently brought up a speculative idea, namely that the dark sector interaction might be probed by measuring the alignment between galaxy and matter distributions in triaxial clusters. In $\Lambda \mathrm{CDM}$ cosmology, the spatial correlations of the large-scale tidal fields yield the strong alignments of the satellite galaxies in the clusters with the cluster dark matter distributions (e.g., Altay et al. 2006). Lee (2010) claimed that the alignment of the cluster galaxies with the underlying $\mathrm{CDM}$ distribution would be weaker in the $\mathrm{cDE}$ models than in $\Lambda \mathrm{CDM}$ since the spatial correlations of the tidal fields inside the clusters would be less strong due to the existence of a fifth force. If this is really the case, the alignment between galaxy and matter distributions in triaxial clusters would provide a unique probe of the $\mathrm{cDE}$ scenarios, especially because the alignment angle is insensitive to the other cosmological parameters.

To back up the speculative idea of Lee (2010), it is essential to examine numerically whether or not the cDE scalar field truly makes a detectable difference on the cluster galaxymatter alignment. The goal of this Paper is to use high-resolution N-body simulations to study how the alignment between satellites and the underlying dark matter distribution in galaxy clusters changes in cDE models as compared to $\Lambda$ CDM (Komatsu et al. 2010). 


\section{SIMULATIONS AND DATA ANALYSIS}

The dark interaction between the DE scalar field $\phi$ and CDM which characterizes cDE models affects the growth of cosmic structures in a manifold way. First of all, the background expansion history of the universe is changed with respect to the standard $\Lambda \mathrm{CDM}$ evolution by the dynamical nature of the DE scalar field $\phi$. Furthermore, the mass of CDM particles changes in time due to the exchange of energy with the DE sector, and the coupling between the two fields determines a new long-range fifth force between CDM particles and an effective "modified inertia" in the form of a velocity-dependent acceleration. These features can be expressed by a modified acceleration equation for CDM particles in the Newtonian limit:

$$
\dot{\vec{v}}_{i}=\beta(\phi) \frac{\dot{\phi}}{M_{\mathrm{Pl}}} \vec{v}_{i}+\sum_{j \neq i} \frac{G\left[1+2 \beta^{2}(\phi)\right] m_{j}(\phi) \vec{r}_{i j}}{\left|\vec{r}_{i j}\right|^{3}},
$$

where the reduced Planck mass is defined as $M_{\mathrm{Pl}} \equiv 1 / \sqrt{8 \pi G}, \beta(\phi)$ is the coupling strength, $\vec{v}_{i}$ is the velocity of the $i$-th particle, $\vec{r}_{i j}$ is the vector distance between the $i$-th and the $j$-th particles, and the sum extends to all the CDM particles in the universe. The first term on the right-hand side of Equation 11 represents the "modified inertia", while the $\left[1+2 \beta^{2}(\phi)\right]$ factor that multiplies the gravitational constant is the "fifth-force". All these effects have been studied in the literature (see e.g. Baldi 2010a, b) - to which we refer the interested reader for a more detailed explanation of the $\mathrm{cDE}$ effects - and need to be properly included in N-body algorithms in order to consistently evolve the growth of density perturbations in these scenarios.

In the present work, we rely on the numerical implementation of cDE models in the TreePM cosmological N-body code GADGET-2 Springel 2005) presented in Baldi et al. (2010), with which we run a series of cosmological simulations for a fiducial $\Lambda$ CDM cosmology with WMAP7 parameters and two cDE scenarios with the same background parameters and with linear density perturbations normalized at the present time $z=0$. Our cDE models consist of one constant coupling scenario with $\beta=0.2$ (the EXP005 model of Baldi \& Pettorino (2010)) and one variable coupling model with $\beta=0.4 \cdot e^{-2 \phi / M_{\mathrm{Pl}}}$ (the EXP010e2 model of Baldi (2010a)). Notice that the coupling definition assumed in this work is different from some of the literature and our values of the coupling are $\sqrt{2 / 3}$ smaller than the ones quoted in e.g. Amendola (2000). For these three different cosmologies we run a CDM simulation on a cosmological box of 100 comoving $h^{-1} \mathrm{Mpc}$ aside, with $512^{3}$ particles. This corresponds to a space resolution of $\epsilon_{s}=4 h^{-1} \mathrm{kpc}$ and to a mass resolution at $z=0$ of $m=5.6 \times 10^{8} h^{-1} M_{\odot}$. We then link CDM particles into groups with a friends-of-friends procedure and for each main halo we identify all the bound substructures by means of the SUBFIND algorithm (Springel et al. 2001). Let us remind that we use the same initial ran- 
dom field in all our simulations. This allows us the possibility of a one-to-one comparison between different models.

In order to compare the results of our numerical simulations with observations we need to assign to each (sub)halo a stellar mass. For this purpose we use the recently derived stellar-to-halo mass relationship presented in Moster et al. (2010). In this paper the authors give a series of simple fitting formulae to derive the stellar mass of the galaxy hosted by a given dark matter halo as a function of the virial mass (for central galaxies) or as a function of the maximum mass over the halo's history (for satellite galaxies). For all our substructures we therefore build a full merger tree and we assign to each subhalo a stellar mass based on the latter criterion by using the formulae of Moster et al. (2010).

However, these formulae have been derived by comparing the observed galaxy luminosity function with the halo mass function in a $\Lambda$ CDM universe. This implies that we cannot directly apply them in the case of cDE models. In order to overcome this issue, we first find a function $f(M, z)$ able to map the cDE mass function onto the $\Lambda$ CDM one and then we apply the Moster et al. (2010) fitting equations. The CDM density and the location of the 30 most luminous substructures inside two virial radii for the same massive cluster within the three different cosmologies under investigation are plotted in the three panels of Figure 1, where a stronger tendency of alignment between the CDM distribution and the selected substructures in the $\Lambda$ CDM model as compared to cDE scenarios is already visible.

\section{ALIGNMENT BETWEEN GALAXY AND MATTER DISTRIBUTIONS IN CLUSTERS}

From each cluster catalog obtained from the simulations for the $\Lambda$ CDM and cDE cosmologies, we select those cluster halos which have five or more galaxies within some cut-off radius $r_{c}\left(\leq R_{\text {vir }}\right)$ from the halo center. Then, we measure the alignment angles between the galaxy and dark matter distributions of each selected cluster halo through the following procedure. First, the inertia tensor of the galaxy distribution of each selected cluster halo, $\left(I_{i j}^{G}\right)$, is evaluated as

$$
I_{i j}^{G}=\sum_{\beta} m_{*, \beta} x_{\beta, i} x_{\beta, j},
$$

where $m_{*, \beta}$ is the stellar mass of the $\beta$-th galaxy and $\left(x_{\beta, i}\right)$ is the position vector of the $\beta$-th satellite galaxy relative to the center of the stellar mass of the galaxy distribution. Weighting the satellites by stellar mass is important for a comparison with observations since in practice the shapes of galaxy distributions are often obtained from the luminosity-weighted satellites. Finding the unit eigenvector of $\left(I_{i j}^{G}\right)$ corresponding to the largest eigenvalue, we determine 
the major axis, $\mathbf{e}_{G}$, of the galaxy distribution of each selected cluster halo.

Similarly, we evaluate the inertia tensor, $\left(I_{i j}^{M}\right)$, of the CDM distribution of each selected cluster halo as

$$
I_{i j}^{M}=\sum_{\alpha} x_{\alpha, i} x_{\alpha, j}
$$

where $\left(x_{\alpha, i}\right)$ is the position vector of the $\alpha$-th dark matter particle relative to the halo center. The summation in Equation (3) goes over all dark matter particles located within the halo virial radius $R_{v i r}$. Finding the unit eigenvector of $\left(I_{i j}^{M}\right)$ corresponding to the largest eigenvalue, we determine the major axis, $\mathbf{e}_{M}$, of the CDM distribution of each selected cluster halo. The alignment angle, $\theta$, between dark matter and galaxy distributions of each selected halo is now calculated as $\cos \theta=\left|\mathbf{e}_{M} \cdot \mathbf{e}_{G}\right|$ where $\cos \theta$ is in the range of $[0,1]$. Binning the values of $\cos \theta$ and counting the number of the halos belonging to each $\cos \theta$-bin, we derive the probability density distribution, $p(\cos \theta)$, for the $\Lambda \mathrm{CDM}$ and $\mathrm{cDE}$ cosmologies, separately. When the cut-off radius $r_{c}$ for the satellite galaxies is set at $0.8 R_{\text {vir }}$ (see Figure 4), the total number of the selected cluster halos which have five or more galaxies within $r_{c}$ is found to be 186 and 116 for the $\Lambda \mathrm{CDM}$ and cDE cosmologies, respectively.

Before making a comparison between the probability distributions of $\cos \theta$ from the two cosmologies, it is worth examining how robust our measurement of $\cos \theta$ is. If the dark matter distribution of a given halo is spherically symmetric, then the determination of the major principal axis of its inertia tensor would be quite ambiguous. Therefore, the robustness of the measurement of $\cos \theta$ of a given halo critically depends on how spherical its dark matter distribution is. The sphericity of a given halo is conventionally defined as $S \equiv \sqrt{u_{3} / u_{1}}$, where $u_{1}$ and $u_{3}$ are the largest and the smallest eigenvalues of the inertia tensor of the dark matter distribution, respectively. Using this definition, we measure the sphericity of each selected halo for both cosmologies. Figure 2 plots the number distribution of the selected halos versus their sphericity for $\mathrm{cDE}$ and $\Lambda \mathrm{CDM}$ cosmologies as solid and dashed histogram, respectively. As it can be seen, for the case of cDE there is no halo whose sphericity is larger than 0.9 , while for $\Lambda$ CDM there is only one halo whose sphericity exceeds 0.9 . Thus, our measurement of $\cos \theta$ is robust, free from the ambiguity caused by the spherically symmetric dark matter distribution.

Given that our samples of the halos for both cosmologies are not large enough to be fully representative of the true parent populations, we perform the bootstrap error analysis to estimate the uncertainties in the measurement of $p(\cos \theta)$ (Wall \& Jenkins 2003). We first construct 1000 bootstrap resamples and measure $p(\cos \theta)$ from each resample. The bootstrap error, $\sigma_{b}$, at each bin is then calculated as one standard deviation among the 1000 remeasurements, $\sigma_{b} \equiv\left\langle\left[p\left(\cos \theta_{i}\right)-\left\langle p\left(\cos \theta_{i}\right)\right\rangle\right]^{2}\right\rangle^{1 / 2}$, where the ensemble average is taken over the 1000 resamples and $\left\langle p\left(\cos \theta_{i}\right)\right\rangle$ represents the bootstrap mean value at the $i$-th bin. The 
top panel of Figure 3 plots the resulting $p(\cos \theta)$ with the bootstrap errors for $\mathrm{cDE}$ and $\Lambda \mathrm{CDM}$ cosmologies as square and triangle dots, respectively. For this plot, the cut-off radius $r_{c}$ for the satellite galaxies is set at $0.8 R_{v i r}$.

As shown in Figure 3, the probability density distribution increases with $\cos \theta$ in both cosmologies, which indicates that the major axes of the galaxy and dark matter distributions in clusters tend to be strongly aligned. However, we note a difference between the two cosmologies: $p(\cos \theta)$ increases less rapidly in the cDE model. At the fourth $\cos \theta$-bin (corresponding to $0.6 \leq \cos \theta \leq 0.8)$, the difference between the two models exceeds $3 \sigma_{b}$. The bottom panel of Figure 3 plots the ratio between the two probability density distributions: $p_{c D E}(\cos \theta) / p_{\Lambda C D M}(\cos \theta)$, which demonstrates clearly that the ratio deviates from unity and the degree of the deviation reaches the maximum at the fourth bin.

Now that we find a difference between the two cosmologies in the tendency of the cluster galaxy-matter alignment, we would like to investigate how the result depends on the cutoff radius $r_{c}$. In fact, it is naturally expected that as $r_{c}$ decreases the alignment tendency would decrease. The major axes of the dark matter distribution are determined at $R_{v i r}$ while the major axes of the galaxy distribution are measured at $r_{c}$. Therefore, the difference $R_{v i r}-r_{c}$ represents the distance by which the inner and outer tidal fields are separated. As $r_{c}$ decreases, the spatial correlations between the two tidal fields would decrease, which would be manifested by the decrease of the strength of the alignment between the major axes of the galaxy and matter distributions in clusters.

Repeating the whole process described above, we calculate the probability density at the fourth bin, $p(0.6<\cos \theta<0.8)$, for five different choices for $r_{c}$. The top panel of Figure 4 plots the result for $\mathrm{cDE}$ and $\Lambda \mathrm{CDM}$ cosmologies as square and triangle dots, respectively. The bottom panel of Figure 4 plots the ratio of the square points (cDE) to the triangle points $(\Lambda \mathrm{CDM})$. Figure 4 reveals that in the $\mathrm{cDE}$ scenario the probability density, $p(0.6<$ $\cos \theta<0.8)$, decreases more rapidly as $r_{c}$ decreases. This result may be interpreted as the effect of coupled dark energy on the spatial correlations of the tidal fields inside the cluster halos. Due to the coupling between dark matter and dark energy, there is an additional tidal force exerted on the galaxies inside the clusters, which plays a role of reducing further the correlations of galaxy distributions with matter distributions. Furthermore, the velocitydependent acceleration arising as a consequence of the "modified inertia" described by the first term on the right-hand side of Equation (1) also contributes to an effective modification of the internal tidal field of the cluster experienced by the substructures since it determines an effective extra force along the direction of motion of the satellite halos.

To make a direct comparison with observations, it may be more useful to find the probability density distribution of the two dimensional projected alignment angles, $\theta_{2 \mathrm{~d}}$. For each 
cosmological model, we project $\mathbf{e}_{G}$ and $\mathbf{e}_{M}$ of each selected halo cluster onto the xy, yz and zx planes and calculate the two dimensional alignment angle $\theta_{2 \mathrm{~d}}$ between the projected major axes of dark matter and galaxy distributions for all three projection cases and determine the probability density distribution, $p\left(\theta_{2 d}\right)$, by taking the average over the three cases. To estimate the uncertainties in the measurement of $p\left(\theta_{2 d}\right)$, we also perform the boostrap error analysis. The top panel of Figure 5 plots the $p\left(\theta_{2 d}\right)$ with the bootstrap errors for cDE and $\Lambda \mathrm{CDM}$ cosmologies as square and triangle dots, respectively. As one can see, the two dimensional projection does not dilute the difference in the alignment tendency between the two models. The probability density distribution, $p\left(\theta_{2 \mathrm{~d}}\right)$, decreases less rapidly as $\theta_{2 \mathrm{~d}}$ increases in the $\mathrm{cDE}$ model than in the $\Lambda \mathrm{CDM}$ model. Note that for a three dimensional distribution, it is $p(\cos \theta)$ that is expected to be uniformly distributed in case of no alignment while for the two dimensional case, it is $p\left(\theta_{2 \mathrm{~d}}\right)$.

To quantify the statistical significance of the difference in the galaxy-matter alignments between $\Lambda \mathrm{CDM}$ and $\mathrm{cDE}$ cosmologies, we perform a Wald test for the ratio, $\xi \equiv$ $p_{c D E}(\cos \theta) / p_{C D E \Lambda C D M}(\cos \theta)$. In the null hypothesis $H_{N}$ that the two probability density distributions shown in Figures 3 and 5 are in fact from the same parent population, the expectation value of $\xi$ is unity at all bins. Taking into account the possible cross-correlations of $p(\cos \theta)$ between the different bins, we calculate the generalized $\chi^{2}$ defined in terms of the full covariance matrix as

$$
\chi^{2} \equiv\left(\xi_{i}-1\right) C_{i j}^{-1}\left(\xi_{j}-1\right) .
$$

Here $C_{i j}^{-1}$ is the inverse of the covariance matrix, $C_{i j} \equiv\left\langle\left(\xi_{i}-\bar{\xi}_{i}\right)\left(\xi_{j}-\bar{\xi}_{j}\right)\right\rangle$, where the ensemble average is taken over the 1000 bootstrap resamples and $\bar{\xi}_{i}$ denotes the mean value of $\xi$ averaged over the bootstrap resamples at the $i$-th bin. In Equation (44),$\left(\xi_{i}-1\right)$ expresses how much the numerical result of $\xi$ at the $i$-th bin deviates from the expectation value of unity. We find $\chi^{2}=15.08$ and 14.97 for the three and two dimensional case, respectively. Since the number of degrees of freedom for $\chi^{2}$ is 5 , its value corresponds to the null hypothesis being rejected at the $98.9 \%$ confidence level for both cases.

\section{DISCUSSION AND CONCLUSIONS}

Using data from high-resolution N-body simulations, we have shown that the $\Lambda$ CDM and $\mathrm{cDE}$ cosmologies yield different strengths of the alignment between satellite galaxy and matter distributions in triaxial clusters. As speculated by Lee (2010), it is found that the alignment is less strong in cDE cosmologies than in the standard $\Lambda$ CDM scenario. The null hypothesis that the two cosmologies give the same alignment tendency is rejected at the $98.9 \%$ confidence level through a Wald test based on the generalized $\chi^{2}$ statistics. 
The differences in the satellite distributions between $\Lambda \mathrm{CDM}$ and cDE models may be understood as follows. There are two possible mechanisms for explaining the alignments between the dark matter and galaxy distributions (Altay et al. 2006). The first one is the spatial correlations between the large scale external tidal fields and the internal tidal fields due to the cluster potentials which affect he non-spherical shapes of dark matter components of galaxy clusters and the anisotropic distributions of their satellite galaxies, respectively. The second one is the filamentary merging/accretion of matter and galaxies along the large-scale filaments, which also induce the alignments between the dark matter and galaxy distributions in clusters.

In the $\Lambda$ CDM cosmology, the tidal fields evolve nonlinearly only via gravity. Whereas in the cDE cosmology the coupling between dark energy and dark matter generate additional (more isotropic) tidal effects in the clusters, which weaken the spatial correlations between the external and internal tidal fields and also redistribute previously accreted satellites that were initially in a flattened distribution. Furthermore, the "modified inertia" characterizing cDE models would contribute to an effective enhancement of the internal cluster tidal field.

Recently Oguri et al. (2010) compared the observed distribution of satellite galaxies in clusters with the shape of the cluster dark matter distribution determined from weak lensing, finding a very weak alignment (Lee 2010). Due to the small sample (composed of only 13 clusters), it is difficult to say at the moment whether or not this observational signal supports the cDE scenario. In any case, a crucial implication of our results is that the alignment between galaxy and matter distributions in clusters is in principle a unique probe of dark sector interactions.

We thank an anonymous referee for helpful suggestions. M.B. is supported by the DFG Cluster of Excellence "Origin and Structure of the Universe". J.L. acknowledges the support from the National Research Foundation of Korea (NRF) grant funded by the Korea government (MEST, No.2010-0007819) and partial support provided by the National Research Foundation of Korea to the Center for Galaxy Evolution Research. All the numerical simulations have been performed on the VIP cluster at the RZG computing center in Garching.

\section{REFERENCES}

Abdalla, E., Abramo, L. R., Sodré, L., \& Wang, B. 2009, Physics Letters B, 673, 107

Altay, G., Colberg, J. M., \& Croft, R. A. C. 2006, MNRAS, 370, 1422 
Amendola, L. 2000, Phys. Rev. D, 62, 043511

Amendola L., 2004, Phys. Rev. D, 69, 103524

Baldi, M., MNRAS, in press arXiv:1005.2188]

Baldi, M., arXiv:1012.0002

Baldi M., Pettorino V., MNRAS, in press, arXiv:1006.3761]

Baldi, M., \& Viel, M. 2010, MNRAS, 409, L89

Baldi, M., Pettorino, V., Robbers, G., \& Springel, V. 2010, MNRAS, 403, 1684

Farrar, G. R., \& Rosen, R. A. 2007, Physical Review Letters, 98, 171302

Keselman, J. A., Nusser, A., \& Peebles, P. J. E. 2009, Phys. Rev. D, 80, 063517

Komatsu, E., et al. 2010, ApJ, in press

Lee, J. 2010, arXiv:1008.4620

Li, B. \& Barrow, J., arXiv:1005.4231

Macciò, A. V., Quercellini, C., Mainini, R., Amendola, L., \& Bonometto, S. A. 2004, Phys. Rev. D, 69, 123516

Mainini, R. \& Bonometto, S., Phys. Rev. D, 74, 043504

Moster, B., et al. 2010, ApJ, 710, 903

Oguri, M., Takada, M., Okabe, N., \& Smith, G. P. 2010, MNRAS, 405, 2215

Pettorino V., Baccigalupi C., 2008, Phys. Rev. D, 77, 103003

Ratra B., Peebles P. J. E., 1988, Phys. Rev. D, D37, 3406

Springel, V., MNRAS, 364, 1105

Springel, V., et al. 2001, MNRAS, 328, 726

Wall, J. V. \& Jenkins, C. R. 2003, Practical Statistics for Astronomers (Cambridge: Cambridge Univ. Press)

Wetterich, C., Nucl. Phys., B302, 668

Wetterich C., 1995, Astron. Astrophys., 301, 321 
Wintergerst N., Pettorino V., 2010, Phys. Rev. D, 82, 103516

This preprint was prepared with the AAS LATEX macros v5.2. 

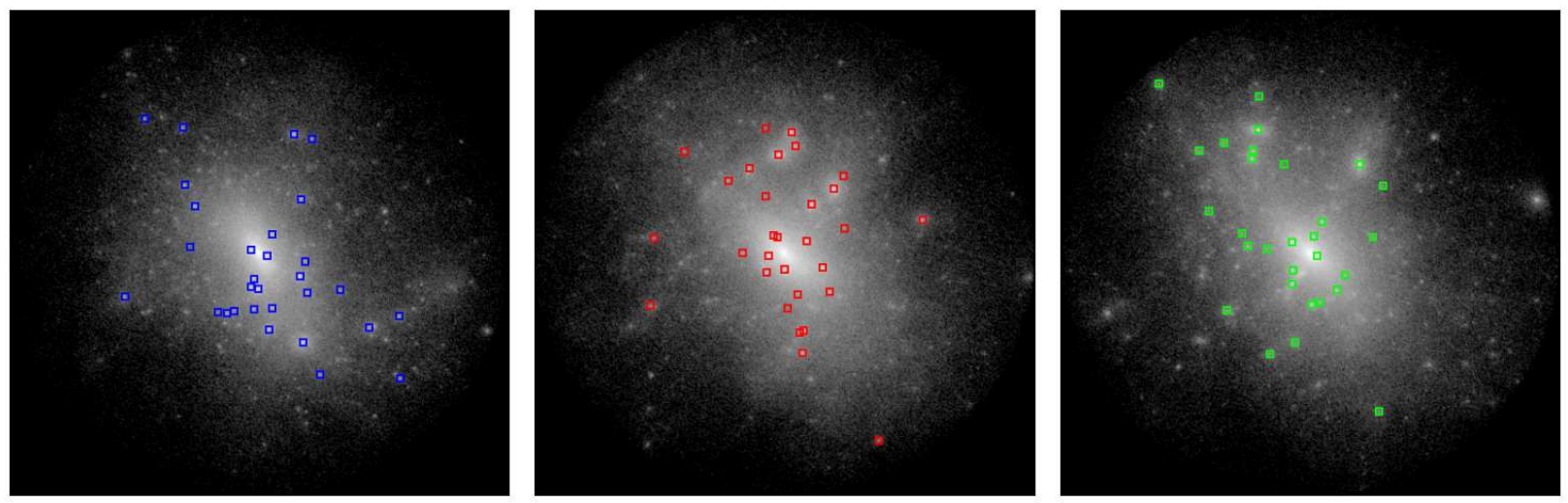

Fig. 1. - CDM projected density (grey scale) and location of the 30 most luminous substructures (colored squares) within $R_{200}$ for the most massive cluster formed in our simulated

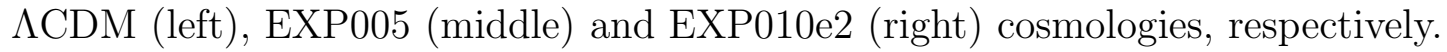




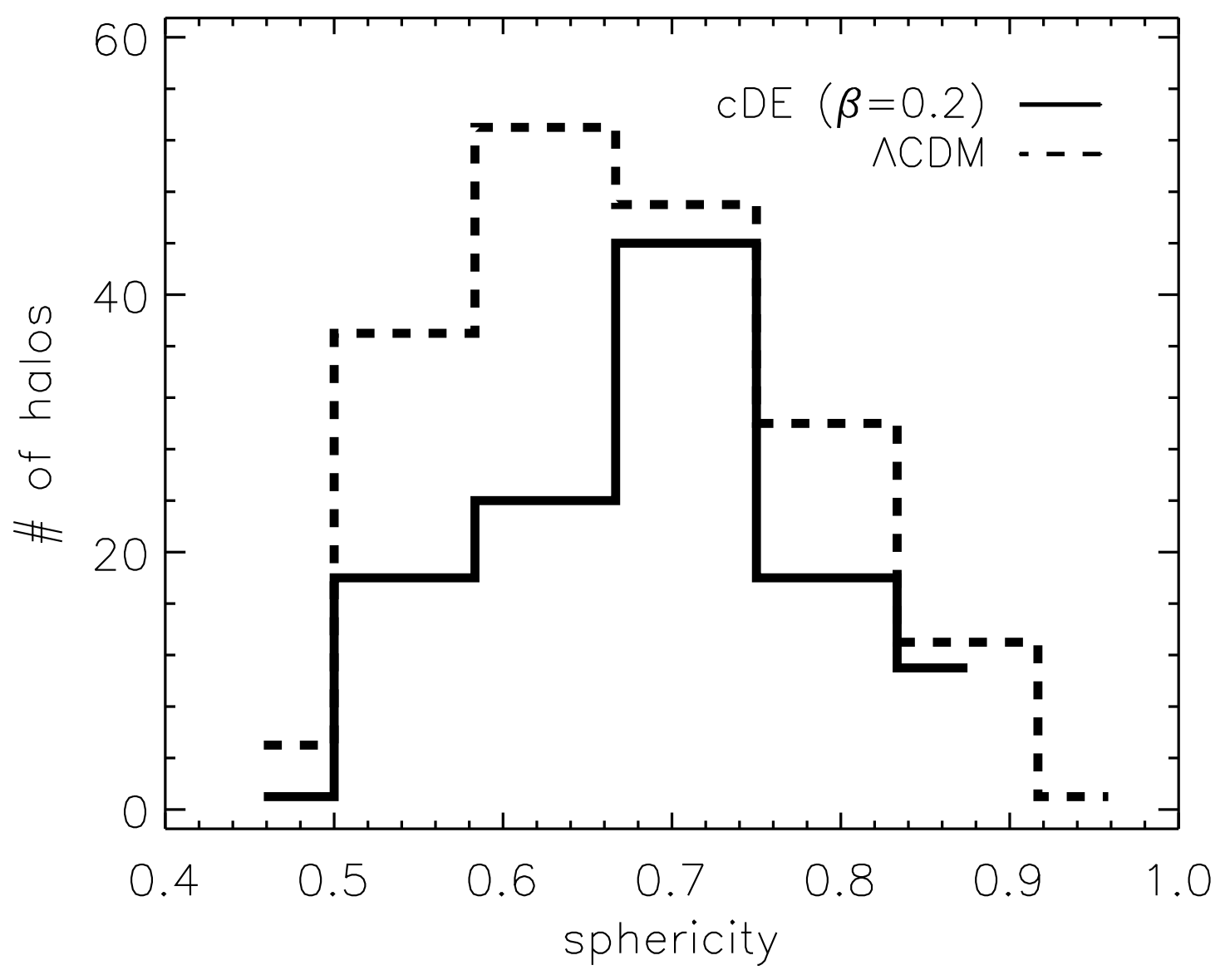

Fig. 2.- Number distributions of the selected halos as a function of the sphericity of their dark matter distribution for two different cosmologies:cDE (solid histogram) and $\Lambda$ CDM (dashed histogram). 


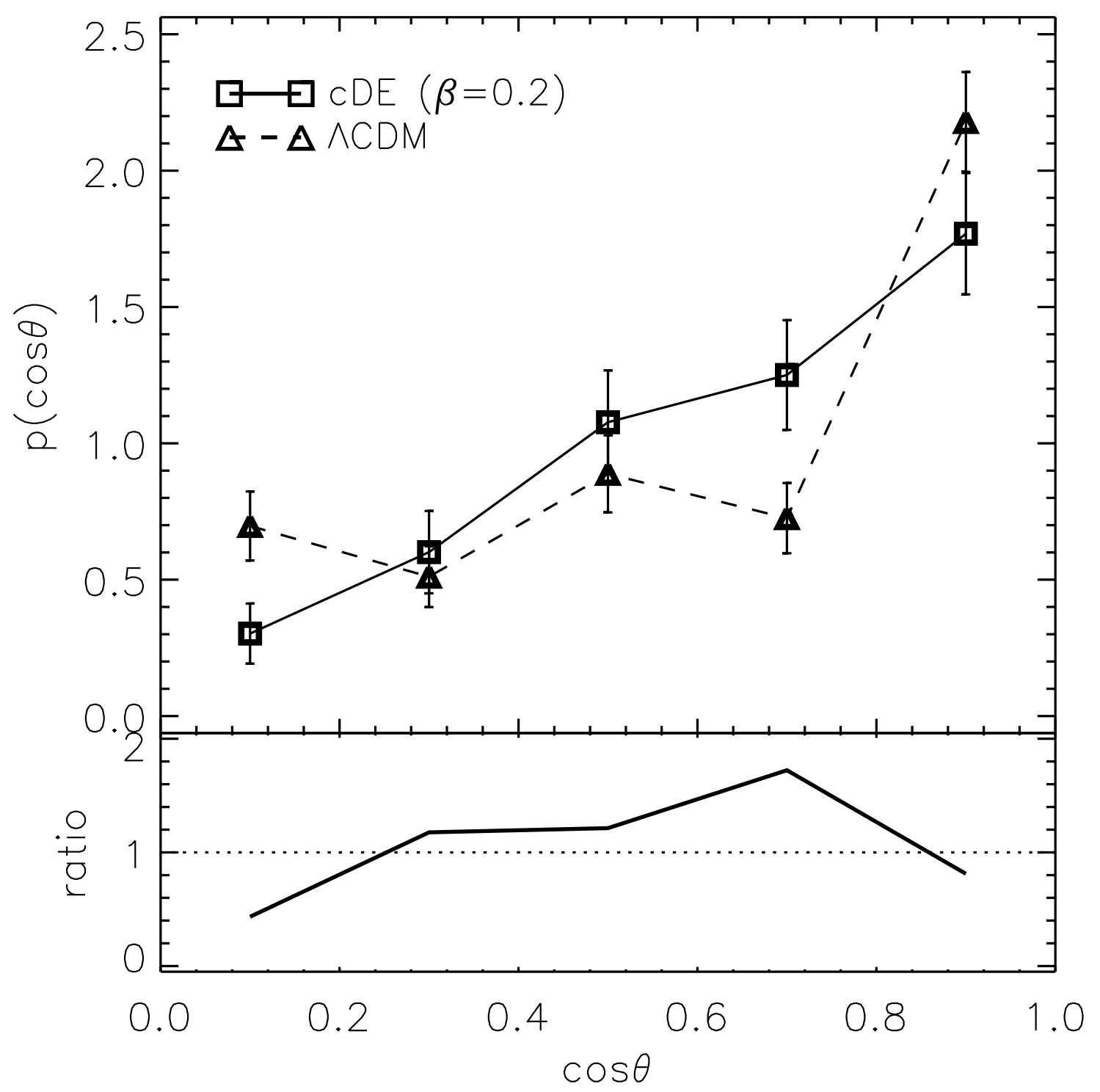

Fig. 3.- Probability density distribution of the cosines of the angles between the major axes of dark matter and galaxy distributions of the simulated clusters for two different cosmologies: $\mathrm{cDE}$ (solid line+square dots) and $\Lambda \mathrm{CDM}$ (dashed line+triangle dots) in the top panel. The errors represent the $1 \sigma$ scatter among the 1000 bootstrap resamples. The ratios of the square points to the triangle points are plotted as solid line in the bottom panel. 


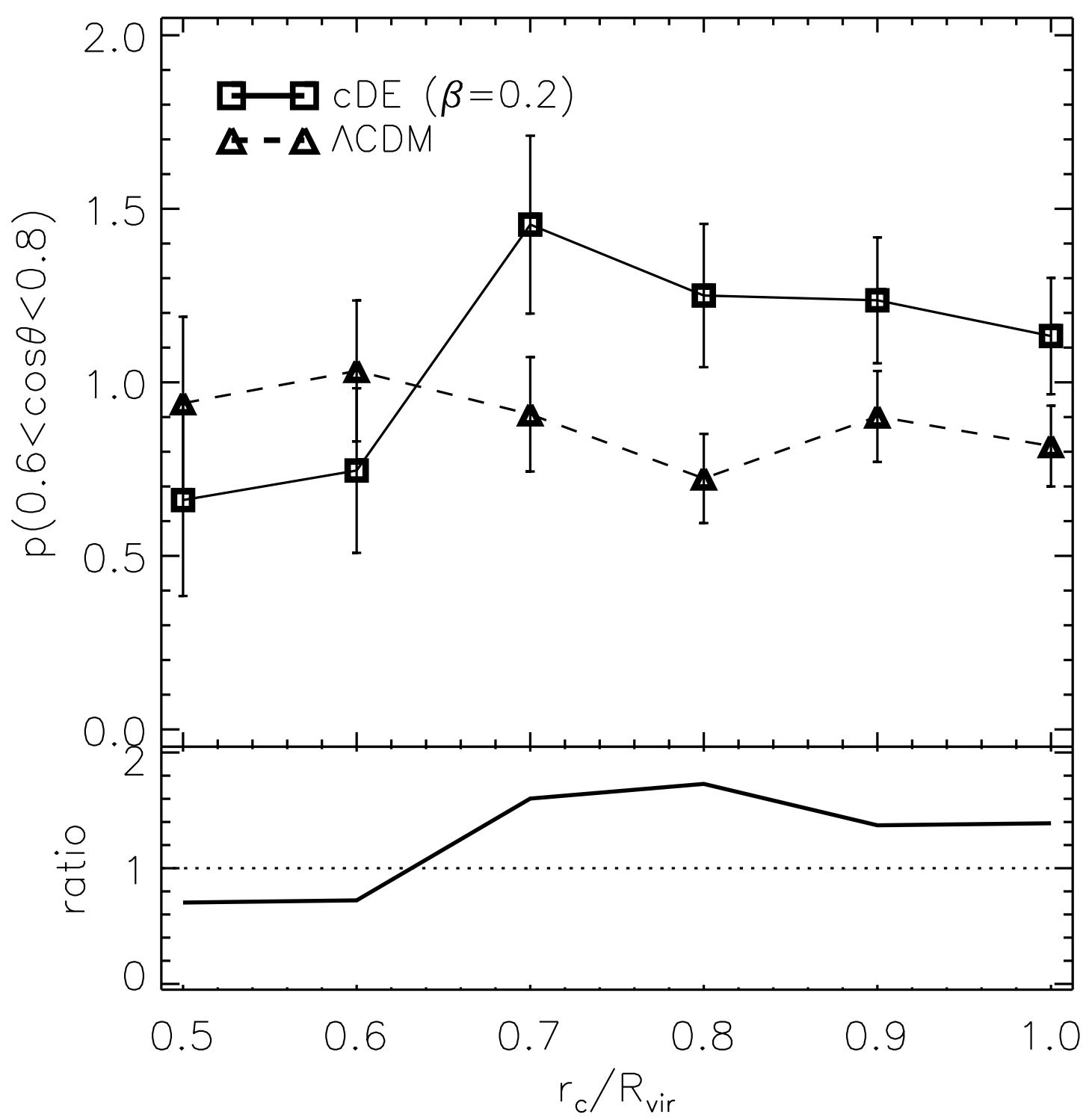

Fig. 4.- Probability density that the cosines of the angles between the major axes of dark matter and galaxy distributions of the simulated clusters are in the range of $(0.6,0.8)$ with the bootstrap errors as a function of the cut-off radius of the satellite galaxies $r_{c}$ for two cosmological models: cDE (solid line+ square dots) and $\Lambda$ CDM (dashed line+triangle dots). in the top panel The ratios of the square points to the triangle points at each bin are plotted as solid line in the bottom panel. 


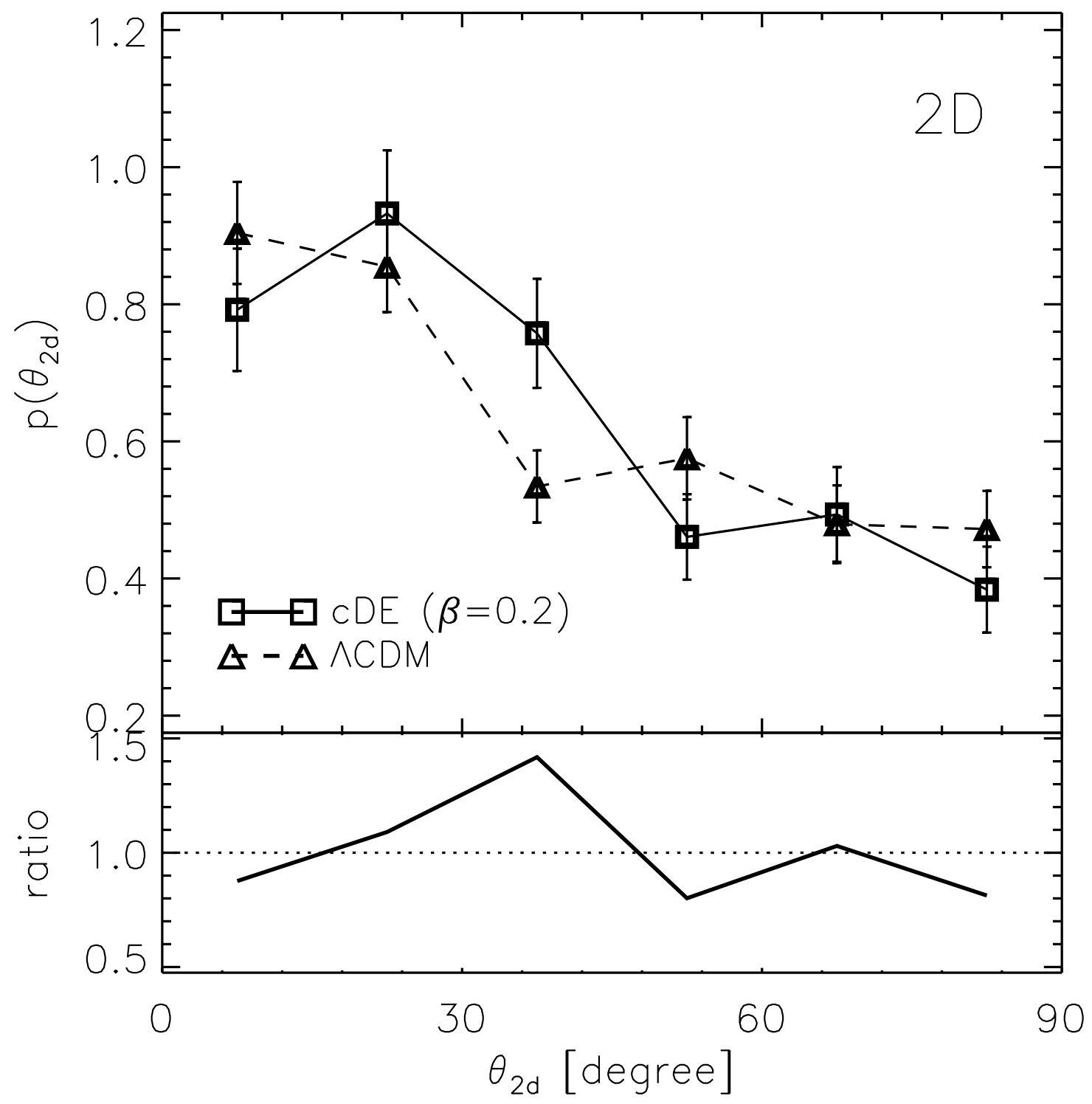

Fig. 5.- Probability density distribution of the angles between the two dimensional projected major axes of dark matter and galaxy distributions with the bootstrap errors for two different cosmologies: $\mathrm{cDE}$ (solid line+square dots) and $\Lambda \mathrm{CDM}$ (dashed line+triangle dots). The ratios of the square points to the triangle points are plotted as solid line in the bottom panel. 